\title{
ADDITIVE RESULTS FOR THE GENERALIZED DRAZIN INVERSE
}

\author{
DRAGAN S. DJORDJEVIĆ and YIMIN WEI
}

(Received 2 February 2001; revised 6 August 2001)

Communicated by A. Pryde

\begin{abstract}
Additive perturbation results for the generalized Drazin inverse of Banach space operators are presented. Precisely, if $A^{d}$ denotes the generalized Drazin inverse of a bounded linear operator $A$ on an arbitrary complex Banach space, then in some special cases $(A+B)^{d}$ is computed in terms of $A^{d}$ and $B^{d}$. Thus, recent results of Hartwig, Wang and Wei (Linear Algebra Appl. 322 (2001), 207-217) are extended to infinite dimensional settings with simplified proofs.
\end{abstract}

2000 Mathematics subject classification: primary 47A05, 47A55, 15 A09.

Keywords and phrases: generalized Drazin inverse, perturbations, additive results.

\section{Introduction}

Let $X$ denote an arbitrary complex Banach space and $\mathscr{L}(X)$ denote the Banach algebra of all bounded operators on $X$. If $A \in \mathscr{L}(X)$, then $\mathscr{R}(A), \mathscr{N}(A)$ and $\sigma(A)$, respectively, denote the range, kernel and spectrum of $A$.

If $S$ is a subset of the complex plane, then acc $S$ and iso $S$, respectively, denote the set of all points of accumulation and the set of all isolated points of $S$.

If $0 \notin \operatorname{acc} \sigma(A)$, then the function $z \mapsto f(z)$ can be defined as $f(z)=0$ in a neighbourhood of 0 and $f(z)=1 / z$ in a neighbourhood of $\sigma(A) \backslash\{0\}$. Then $z \mapsto f(z)$ is regular in a neighbourhood of $\sigma(A)$ and the generalized Drazin inverse

The first author was supported by the Project No. 1232 of the Ministry of Science, Technologies and Development, Republic of Serbia.

The second author was supported by the National Natural Science Foundation of China, project 19901006. Partial work was finished when the second author visited Harvard University and was supported by the China Scholarship Council.

(C) 2002 Australian Mathematical Society 1446-7887/2000\$A2.00+0.00 
of $A$ is defined using the functional calculus as $A^{d}=f(A)$ (see the well-known Koliha's paper [14]). Notice that $A^{d} A=A A^{d}, A^{d} A A^{d}=A^{d}$ and that $A A^{d} A-A$ is quasinilpotent. We say that $A \in \mathscr{L}(X)$ is GD-invertible if $0 \notin \operatorname{acc} \sigma(A)$. If $A$ is GD-invertible, then the spectral idempotent $P$ of $A$ corresponding to $\{0\}$ is given by $P=I-A A^{d}$. The matrix form of $A$ with respect to the decomposition $X=\mathscr{N}(P) \oplus \mathscr{R}(P)$ is given by $A=\left[\begin{array}{cc}A_{1} & 0 \\ 0 & A_{2}\end{array}\right]$, where $A_{1}$ is invertible and $A_{2}$ is quasinilpotent. We can also write

$$
A=C_{A}+Q_{A}, \quad C_{A}=\left[\begin{array}{cc}
A_{1} & 0 \\
0 & 0
\end{array}\right], \quad Q_{A}=\left[\begin{array}{cc}
0 & 0 \\
0 & A_{2}
\end{array}\right],
$$

and $A=C_{A}+Q_{A}$ is known as the core-quasinilpotent decomposition of $A$.

If $A$ is GD-invertible, then the resolvent function $z \mapsto(z I-A)^{-1}$ is defined in a punctured neighbourhood of $\{0\}$. If $z=0$ is a pole of the resolvent function of $A$, then the order of this pole is known as the Drazin index of $A$, denoted by ind $(A)$. In this case we say that $A$ is D-invertible. If ind $(A)=k$, then $A^{d}$ reduces to the ordinary Drazin inverse of $A$, denoted by $A^{D}$. Thus, $A^{D}$ is the unique operator satisfying conditions

$$
A^{m+1} A^{D}=A^{m}, \quad A^{D} A A^{D}=A^{D}, \quad A A^{D}=A^{D} A,
$$

and $m$ is the least integer ind $(A)$ such that (2) is satisfied. Now the core-quasinilpotent decomposition reduces to the core-nilpotent decomposition. Precisely, $\operatorname{ind}(A)=k$ holds if and only if $k$ is the least integer (if it exists) such that $A_{2}^{k}=0$ (recall notations from (1)).

If ind $(A) \leq 1$, then $A^{D}$ is known as the group inverse of $A$, denoted by $A^{*}$. Also, $\operatorname{ind}(A)=0$ if and only if $A$ is invertible and in this case $A^{D}=A^{-1}$. Notice that $\operatorname{ind}\left(C_{A}\right) \leq 1$ always holds and $C_{A}^{*}=\left[\begin{array}{cc}A_{1}^{-1} & 0 \\ 0 & 0\end{array}\right]$ (see (1)).

The Drazin inverse in semigroups and associative rings was first introduced in [7]. The Drazin inverse of complex square matrices was investigated, among other papers and books, in [1] and [2]. A detailed treatment of the Drazin inverse in infinite dimensional spaces is given in [3] and [10]. The generalized Drazin inverse in Banach algebras was introduced in [14]. We mention that an alternative definition of a generalized Drazin inverse in a ring is also given in $[9,10,11]$. These two concepts of a generalized Drazin inverse are equivalent in the case when the ring is actually a complex Banach algebra with a unit.

In this paper we investigate the generalized Drazin inverse $(A+B)^{d}$ in terms of $A^{d}$ and $B^{d}$. Hartwig, Wang and Wei investigated this problem in a finite dimensional case (see [13]). In this paper we generalize their results to infinite dimensional setting, using an alternative approach based on matrix representation of operators relative to a space decomposition. This enables us to simplify the proofs. 


\section{Results}

First we state the following auxiliary result.

LEMMA 2.1. If $P, Q \in \mathscr{L}(X)$ are quasinilpotent and $P Q=0$ or $P Q=Q P$, then $P+Q$ is also quasinilpotent. Hence, $(P+Q)^{d}=0$.

Proof. Case I. Let $P Q=0$ and $\lambda \neq 0$. Then $\lambda-P$ and $\lambda-Q$ are invertible and consequently $\lambda-P-Q=\lambda^{-1}(\lambda-P)(\lambda-Q)$ is invertible.

Case II. Let $P Q=Q P$ and let $\sigma(P, Q)$ denote the Harte joint spectrum of $(P, Q)$. It follows that $\sigma(P, Q)=\{(0,0)\}$. An application of the spectral mapping theorem for this spectrum leads to $\sigma(P+Q)=\{0\}$ (see [10] for details). Thus, the proof is completed.

The following result is a generalization of [13, Lemma 1.1].

THEOREM 2.1. Let $A, B, A+B \in \mathscr{L}(X)$ be GD-invertible and $A B=B A$. Then $(A+B)^{d}=\left(C_{A}+C_{B}\right)^{d}\left[I+\left(C_{A}+C_{B}\right)^{d}\left(Q_{A}+Q_{B}\right)\right]^{-1}$.

Proof. Let $P$ be the spectral idempotent of $A$ corresponding to $\{0\}$. Then $A$ has the matrix form $A=\left[\begin{array}{cc}A_{1} & 0 \\ 0 & A_{2}\end{array}\right]$ with respect to the decomposition $X=\mathscr{N}(P) \oplus \mathscr{R}(P)$, where $A_{1}$ is invertible and $A_{2}$ is quasinilpotent. Now we have $C_{A}=\left[\begin{array}{cc}A_{1} & 0 \\ 0 & 0\end{array}\right]$ and $Q_{A}=\left[\begin{array}{ll}0 & 0 \\ 0 & A_{2}\end{array}\right]$. Since $B$ commutes with $P$, we conclude that $B$ has the form

$$
B=\left[\begin{array}{cc}
B_{1} & 0 \\
0 & B_{2}
\end{array}\right]:\left[\begin{array}{l}
\mathscr{N}(P) \\
\mathscr{R}(P)
\end{array}\right] \rightarrow\left[\begin{array}{l}
\mathscr{N}(P) \\
\mathscr{R}(P)
\end{array}\right]
$$

From $\sigma(B)=\sigma\left(B_{1}\right) \cup \sigma\left(B_{2}\right)$ we get that $B_{1}$ and $B_{2}$ are GD-invertible. From the definition of the generalized Drazin inverse and properties of the functional calculus we get that $B^{d}=\left[\begin{array}{cc}B_{1}^{d} & 0 \\ 0 & B_{2}^{d}\end{array}\right]$, and $C_{B}=\left[\begin{array}{cc}C_{B_{1}} & 0 \\ 0 & C_{B_{2}}\end{array}\right], Q_{B}=\left[\begin{array}{cc}Q_{B_{1}} & 0 \\ 0 & Q_{B_{2}}\end{array}\right]$. From $A B=B A$ we conclude that $A_{i} B_{i}=B_{i} A_{i}$ for $i=1,2$. Let $P_{1}$ be the spectral idempotent of $B_{1}$ in the Banach algebra $\mathscr{L}(\mathscr{N}(P))$ corresponding to $\{0\}$ and let $P_{2}$ be the spectral idempotent of $B_{2}$ in the algebra $\mathscr{L}(\mathscr{R}(P))$ corresponding to $\{0\}$. We have the matrix form of $B_{1}=\left[\begin{array}{cc}B_{3} & 0 \\ 0 & B_{4}\end{array}\right]$ with respect to the decomposition $\mathscr{N}(P)=\mathscr{N}\left(P_{1}\right) \oplus \mathscr{R}\left(P_{1}\right)$, where $B_{3}$ is invertible and $B_{4}$ is quasinilpotent. The matrix form of $B_{2}$ is given as $B_{2}=\left[\begin{array}{cc}B_{5} & 0 \\ 0 & B_{6}\end{array}\right]$, with respect to the decomposition $\mathscr{R}(P)=\mathscr{N}\left(P_{2}\right) \oplus \mathscr{R}\left(P_{2}\right)$, where $B_{5}$ is invertible and $B_{6}$ is quasinilpotent. Since $A_{i}$ commutes with $P_{i}$ for $i=1,2$, we conclude that $A_{1}$ and $A_{2}$, respectively, have the following matrix forms: $A_{1}=\left[\begin{array}{cc}A_{3} & 0 \\ 0 & A_{4}\end{array}\right]$, where $A_{1}$ and $A_{3}$ are invertible, and $A_{2}=\left[\begin{array}{cc}A_{5} & 0 \\ 0 & A_{6}\end{array}\right]$, where $A_{5}$ and $A_{6}$ are quasinilpotent. The last statement follows from the fact $\sigma\left(A_{2}\right)=\sigma\left(A_{5}\right) \cup \sigma\left(A_{6}\right)$. 
Notice that

$$
A+B=\left[\begin{array}{cccc}
A_{3}+B_{3} & 0 & 0 & 0 \\
0 & A_{4}+B_{4} & 0 & 0 \\
0 & 0 & A_{5}+B_{5} & 0 \\
0 & 0 & 0 & A_{6}+B_{6}
\end{array}\right]
$$

Since $0 \notin \operatorname{acc} \sigma(A+B)$ and $\sigma(A+B)=\bigcup_{i=3}^{6} \sigma\left(A_{i}+B_{j}\right)$, we conclude that $A_{i}+B_{i}$ is GD-invertible for all $i=3,4,5,6$. Since $A_{4}$ is invertible and $B_{4}$ is quasinilpotent, we get $A_{4}+B_{4}=A_{4}\left(I+A_{4}^{-1} B_{4}\right)$. Also, $A_{4}$ commutes with $B_{4}$. Hence, $A_{4}^{-1} B_{4}$ is quasinilpotent and $I+A_{4}^{-1} B_{4}$ is invertible. We get that $A_{4}+B_{4}$ is invertible. Analogously, $A_{5}+B_{5}$ is invertible. Finally, $A_{6}+B_{6}$ is a sum of mutually commuting quasinilpotent elements. From Lemma 2.1 it follows that $A_{6}+B_{6}$ is quasinilpotent.

We have $C_{B_{1}}=\left[\begin{array}{cc}B_{3} & 0 \\ 0 & 0\end{array}\right], C_{B_{2}}=\left[\begin{array}{cc}B_{5} & 0 \\ 0 & 0\end{array}\right]$ and

$$
C_{A}+C_{B}=\left[\begin{array}{cccc}
A_{3}+B_{3} & 0 & 0 & 0 \\
0 & A_{4} & 0 & 0 \\
0 & 0 & B_{5} & 0 \\
0 & 0 & 0 & 0
\end{array}\right]
$$

Since $A_{3}+B_{3}$ is GD-invertible and $A_{4}$ and $B_{5}$ are invertible, we conclude that $C_{A}+C_{B}$ is GD-invertible and

$$
\left(C_{A}+C_{B}\right)^{d}=\left[\begin{array}{cccc}
\left(A_{3}+B_{3}\right)^{d} & 0 & 0 & 0 \\
0 & A_{4}^{-1} & 0 & 0 \\
0 & 0 & B_{5}^{-1} & 0 \\
0 & 0 & 0 & 0
\end{array}\right]
$$

We also have

$$
Q_{A}+Q_{B}=\left[\begin{array}{cccc}
0 & 0 & 0 & 0 \\
0 & B_{4} & 0 & 0 \\
0 & 0 & A_{5} & 0 \\
0 & 0 & 0 & A_{6}+B_{6}
\end{array}\right]
$$

and

$$
\left(C_{A}+C_{B}\right)^{d}\left(Q_{A}+Q_{B}\right)=\left[\begin{array}{cccc}
0 & 0 & 0 & 0 \\
0 & A_{4}^{-1} B_{4} & 0 & 0 \\
0 & 0 & B_{5}^{-1} A_{5} & 0 \\
0 & 0 & 0 & 0
\end{array}\right] .
$$


Now it follows that

$$
\left[I+\left(C_{A}+C_{B}\right)^{d}\left(Q_{A}+Q_{B}\right)\right]^{-1}=\left[\begin{array}{cccc}
I & 0 & 0 & 0 \\
0 & \left(I+A_{4}^{-1} B_{4}\right)^{-1} & 0 & 0 \\
0 & 0 & \left(I+A_{5} B_{5}^{-1}\right)^{-1} & 0 \\
0 & 0 & 0 & I
\end{array}\right]
$$

and we easily conclude that

$$
\begin{aligned}
\left(C_{A}+C_{B}\right)^{d}\left[I+\left(C_{A}+C_{B}\right)^{d}\left(Q_{A}+Q_{B}\right)\right] \\
= \\
=\left[\begin{array}{cccc}
\left(A_{3}+B_{3}\right)^{d} & 0 & 0 & 0 \\
0 & \left(A_{4}+B_{4}\right)^{-1} & 0 & 0 \\
0 & 0 & \left(A_{5}+B_{5}\right)^{-1} & 0 \\
0 & 0 & 0 & 0
\end{array}\right]=(A+B)^{d} .
\end{aligned}
$$

Thus, the proof is completed.

Now we consider the non-commutative case. The following result is proved in [6] (see also [12] and [17] for a finite dimensional case).

LEMMA 2.2. If $A \in \mathscr{L}(X)$ and $B \in \mathscr{L}(Y)$ are $\mathrm{GD}$-invertible, $C \in \mathscr{L}(Y, X)$ and $D \in \mathscr{L}(X, Y)$, then $M=\left[\begin{array}{cc}A & C \\ 0 & B\end{array}\right]$ and $N=\left[\begin{array}{ll}A & 0 \\ D & B\end{array}\right]$ are also GD-invertible and $M^{d}=\left[\begin{array}{cc}A^{d} & S \\ 0 & B^{d}\end{array}\right], N^{d}=\left[\begin{array}{cc}A^{d} & 0 \\ R & B^{d}\end{array}\right]$, where

$$
S=\left(A^{d}\right)^{2}\left[\sum_{n=0}^{\infty}\left(A^{d}\right)^{n} C B^{n}\right]\left(I-B B^{d}\right)+\left(I-A A^{d}\right)\left[\sum_{n=0}^{\infty} A^{n} C\left(B^{d}\right)^{n}\right]\left(B^{d}\right)^{2}-A^{d} C B^{d}
$$

and

$$
R=\left(B^{d}\right)^{2}\left[\sum_{n=0}^{\infty}\left(B^{d}\right)^{n} D A^{n}\right]\left(I-A A^{d}\right)+\left(I-B B^{d}\right)\left[\sum_{n=0}^{\infty} B^{n} D\left(A^{d}\right)^{n}\right]\left(A^{d}\right)^{2}-B^{d} D A^{d} .
$$

We need one particular case of our main result.

THEOREM 2.2. If $P, Q \in \mathscr{L}(X)$ are GD-invertible, $Q \in \mathscr{L}(X)$ is quasinilpotent and $P Q=0$, then $P+Q$ is GD-invertible and $(P+Q)^{d}=\sum_{n=0}^{\infty} Q^{n}\left(P^{d}\right)^{n+1}$.

Proof. Since $P$ is GD-invertible, we conclude that $P$ has the matrix form $\left[\begin{array}{cc}P_{1} & 0 \\ 0 & P_{2}\end{array}\right]$, where $P_{1}$ is invertible and $P_{2}$ is quasinilpotent. From $P Q=0$ we conclude that $Q$ has the matrix form $Q=\left[\begin{array}{cc}0 & 0 \\ Q_{1} & Q_{2}\end{array}\right]$, where $P_{2} Q_{1}=0$ and $P_{2} Q_{2}=0$. Since $\{0\}=\sigma(Q)=\{0\} \cup \sigma\left(Q_{2}\right)$, we conclude that $Q_{2}$ is quasinilpotent. Now we have

$$
P+Q=\left[\begin{array}{cc}
P_{1} & 0 \\
Q_{1} & P_{2}+Q_{2}
\end{array}\right]
$$


where $P_{1}$ is invertible and $P_{2}+Q_{2}$ is quasinilpotent (see Lemma 2.1). From Lemma 2.2 we get that

$$
(P+Q)^{d}=\left[\begin{array}{cc}
\cdot P_{1}^{-1} & 0 \\
\sum_{n=0}^{\infty}\left(P_{2}+Q_{2}\right)^{n} Q_{1} P_{1}^{-n-2} & 0
\end{array}\right] .
$$

By induction on $n$ and using $P_{2} Q_{1}=0$ and $P_{2} Q_{2}=0$, we prove that $\left(P_{2}+Q_{2}\right)^{n} Q_{1}=$ $Q_{2}^{n} Q_{1}$ holds for all $n \geq 0$. Hence,

On the other hand,

$$
\sum_{n=0}^{\infty}\left(P_{2}+Q_{2}\right)^{n} Q_{1} P_{1}^{-n-2}=\sum_{n=0}^{\infty} Q_{2}^{n} Q_{1} P_{1}^{-n-2}
$$

$$
\begin{aligned}
\sum_{n=0}^{\infty} Q^{n}\left(P^{d}\right)^{n+1} & =P^{d}+\sum_{n=1}^{\infty}\left[\begin{array}{cc}
0 & 0 \\
Q_{2}^{n-1} Q_{1} & Q_{2}^{n}
\end{array}\right]\left[\begin{array}{cc}
P_{1}^{-n-1} & 0 \\
0 & 0
\end{array}\right] \\
& =\left[\begin{array}{cc}
P_{1}^{-1} & 0 \\
\sum_{n=0}^{\infty} Q_{2}^{n} Q_{1} P_{1}^{-n-2} & 0
\end{array}\right] .
\end{aligned}
$$

Thus, the proof is completed.

The following result is a generalization of [13, Theorem 2.1$]$.

THEOREM 2.3. If $P, Q \in \mathscr{L}(X)$ are GD-invertible and $P Q=0$, then $P+Q$ is GD-invertible and

$$
(P+Q)^{d}=\left(I-Q Q^{d}\right)\left[\sum_{n=0}^{\infty} Q^{n}\left(P^{d}\right)^{n}\right] P^{d}+Q^{d}\left[\sum_{n=0}^{\infty}\left(Q^{d}\right)^{n} P^{n}\right]\left(I-P P^{d}\right) .
$$

PROOF. Since $Q$ is GD-invertible, we can write $Q=\left[\begin{array}{cc}Q_{1} & 0 \\ 0 & Q_{2}\end{array}\right]$, where $Q_{1}$ is invertible and $Q_{2}$ is quasinilpotent. From $P Q=0$ we obtain that $P=\left[\begin{array}{ll}0 & P_{1} \\ 0 & P_{2}\end{array}\right]$, where $P_{1} Q_{2}=0$ and $P_{2} Q_{2}=0$. From $\sigma(P)=\{0\} \cup \sigma\left(P_{2}\right)$ and $0 \notin \operatorname{acc} \sigma(P)$ we conclude that $P_{2}$ is GD-invertible. Now we have

$$
P+Q=\left[\begin{array}{cc}
Q_{1} & P_{1} \\
0 & P_{2}+Q_{2}
\end{array}\right]
$$

From Theorem 2.2 we know that $P_{2}+Q_{2}$ is GD-invertible. Hence, using Lemma 2.2 we get that $P+Q$ is GD-invertible and

where

$$
(P+Q)^{d}=\left[\begin{array}{cc}
Q_{1}^{-1} & S \\
0 & \left(P_{2}+Q_{2}\right)^{d}
\end{array}\right]
$$

$$
S=\left[\sum_{n=0}^{\infty} Q_{1}^{-n-2} P_{1}\left(P_{2}+Q_{2}\right)^{n}\right]\left[I-\left(P_{2}+Q_{2}\right)\left(P_{2}+Q_{2}\right)^{d}\right]-Q_{1}^{-1} P_{1}\left(P_{2}+Q_{2}\right)^{d}
$$


Using $P_{1} Q_{2}=0$ and $P_{2} Q_{2}=0$ we prove $P_{1}\left(P_{2}+Q_{2}\right)^{n}=P_{1} P_{2}^{n}$ for all $n \geq 0$. Now, using Lemma 2.2, Theorem 2.2, and facts $P_{1} Q_{2}=0$ and $P_{2} Q_{2}=0$, we compute

$$
\begin{aligned}
& {\left[\sum_{n=0}^{\infty} Q_{1}^{-n-2} P_{1}\left(P_{2}+Q_{2}\right)^{n}\right]\left[I-\left(P_{2}+Q_{2}\right)\left(P_{2}+Q_{2}\right)^{d}\right] } \\
&=\left[Q_{1}^{-2} P_{1}+\sum_{n=1}^{\infty} Q_{1}^{-n-2} P_{1} P_{2}^{n}\right]\left[I-\left(P_{2}+Q_{2}\right)\left(P_{2}^{d}+\sum_{n=1}^{\infty} Q_{2}^{n}\left(P_{2}^{d}\right)^{n+1}\right)\right] \\
&=\left[Q_{1}^{-2} P_{1}+\sum_{n=1}^{\infty} Q_{1}^{-n-2} P_{1} P_{2}^{n}\right]\left[I-P_{2} P_{2}^{d}-\sum_{n=1}^{\infty} Q_{2}^{n}\left(P_{2}^{d}\right)^{n}\right] \\
&= Q_{1}^{-2} P_{1}\left(I-P_{2} P_{2}^{d}\right)-Q_{1}^{-2} P_{1} \sum_{n=1}^{\infty} Q_{2}^{n}\left(P_{2}^{d}\right)^{n}+\sum_{n=1}^{\infty} Q_{1}^{-n-2} P_{1} P_{2}^{n}\left(I-P_{2} P_{2}^{d}\right) \\
&-\left[\sum_{n=1}^{\infty} Q_{1}^{-n-2} P_{1} P_{2}^{n}\right]\left[\sum_{n=1}^{\infty} Q_{2}^{n}\left(P_{2}^{d}\right)^{n}\right] \\
&= \sum_{n=0}^{\infty} Q_{1}^{-n-2} P_{1} P_{2}^{n}\left(I-P_{2} P_{2}^{d}\right) .
\end{aligned}
$$

Also, using Theorem 2.2 we get

$$
Q_{1}^{-1} P_{1}\left(P_{2}+Q_{2}\right)^{d}=Q_{1}^{-1} P_{1} \sum_{n=0}^{\infty} Q_{2}^{n}\left(P_{2}^{d}\right)^{n+1}=Q_{1}^{-1} P_{1} P_{2}^{d}
$$

On the other hand, notice that from Lemma 2.2 we get $P^{d}=\left[\begin{array}{cc}0 & P_{1}\left(P_{2}^{d}\right)^{2} \\ 0 & P_{2}^{d}\end{array}\right]$. Hence we have

$$
\begin{aligned}
(I-Q & \left.Q^{d}\right)\left[P^{d}+\sum_{n=1}^{\infty} Q^{n}\left(P^{d}\right)^{n+1}\right]+\left[Q^{d}+\sum_{n=1}^{\infty}\left(Q^{d}\right)^{n+1} P^{n}\right]\left(I-P P^{d}\right) \\
= & {\left[\begin{array}{cc}
0 & 0 \\
0 & P_{2}^{d}
\end{array}\right]+\sum_{n=1}^{\infty}\left[\begin{array}{ll}
0 & 0 \\
0 & I
\end{array}\right]\left[\begin{array}{cc}
Q_{1}^{n} & 0 \\
0 & Q_{2}^{n}
\end{array}\right]\left[\begin{array}{cc}
0 & P_{1}\left(P_{2}^{d}\right)^{n+2} \\
0 & \left(P_{2}^{d}\right)^{n+1}
\end{array}\right] } \\
& +\left[\begin{array}{cc}
Q_{1}^{-1} & -Q_{1}^{-1} P_{1} P_{2}^{d} \\
0 & 0
\end{array}\right]+\sum_{n=1}^{\infty}\left[\begin{array}{cc}
0 & Q_{1}^{-n-1} P_{1} P_{2}^{n-1} \\
0 & 0
\end{array}\right]\left[\begin{array}{cc}
I & -P_{1} P_{2}^{d} \\
0 & I-P_{2} P_{2}^{d}
\end{array}\right] \\
= & {\left[\begin{array}{cc}
Q_{1}^{-1} & \sum_{n=1}^{\infty} Q_{1}^{-n-1} P_{1} P_{2}^{n-1}\left(I-P_{2} P_{2}^{d}\right)-Q_{1}^{-1} P_{1} P_{2}^{d} \\
0 & \sum_{n=0}^{\infty} Q_{2}^{n}\left(P_{2}^{d}\right)^{n+1}
\end{array}\right] . }
\end{aligned}
$$

Thus, the proof is completed.

We can also prove the following result, generalizing [13, Corollary 2.2]. 
THEOREM 2.4. Let $A, H \in \mathscr{L}(X)$ and let $A$ be a GD-invertible operator. Let $F \in \mathscr{L}(X)$ be an idempotent commuting with $A$ such that $F H=H$. If $R=(A-H) F$ is GD-invertible, then $A-H$ is GD-invertible and

$$
\begin{aligned}
(A-H)^{d}= & R^{d}-\sum_{n=0}^{\infty}\left(R^{d}\right)^{n+2} H(I-F) A^{n}\left(I-A A^{d}\right) \\
& +\left(I+R^{d} H\right)(I-F) A^{d}-\left(I-R R^{d}\right) \sum_{n=0}^{\infty}(A-H)^{n} H(I-F)\left(A^{d}\right)^{n+2} .
\end{aligned}
$$

PROOF. Since $F^{2}=F$, we have $X=\mathscr{R}(F) \oplus \mathscr{N}(F)$ and $F=\left[\begin{array}{ll}I & 0 \\ 0 & 0\end{array}\right]$ with respect to this decomposition. Operators $A$ and $F$ mutually commute, hence $A=\left[\begin{array}{cc}A_{1} & 0 \\ 0 & A_{2}\end{array}\right]$. From $\sigma(A)=\sigma\left(A_{1}\right) \cup \sigma\left(A_{2}\right)$ we know that $A_{1} \in \mathscr{L}(\mathscr{R}(F))$ and $A_{2} \in \mathscr{L}(\mathscr{N}(F))$ are GD-invertible and $A^{d}=\left[\begin{array}{cc}A_{1}^{d} & 0 \\ 0 & A_{2}^{d}\end{array}\right]$. Since $F H=H$, we conclude that the matrix form of $H$ is given by $H=\left[\begin{array}{cc}H_{1} & H_{2} \\ 0 & 0\end{array}\right]$. Now we have

$$
A-H=\left[\begin{array}{cc}
A_{1}-H_{1} & -H_{2} \\
0 & A_{2}
\end{array}\right] \text {. }
$$

Notice that $R=\left[\begin{array}{cc}A_{1}-H_{1} & 0 \\ 0 & 0\end{array}\right]$ is GD-invertible, implying that $A_{1}-H_{1} \in \mathscr{L}(\mathscr{R}(F))$ is GD-invertible. From Lemma 2.2 it follows that $A-H$ is GD-invertible and

where

$$
(A-H)^{d}=\left[\begin{array}{cc}
\left(A_{1}-H_{1}\right)^{d} & 0 \\
0 & A_{2}^{d}
\end{array}\right]+\left[\begin{array}{ll}
0 & S \\
0 & 0
\end{array}\right],
$$

$$
\begin{aligned}
S= & \left(A_{1}-H_{1}\right)^{d} H_{2} A_{2}^{d}-\sum_{n=0}^{\infty}\left[\left(A_{1}-H_{1}\right)^{d}\right]^{n+2} H_{2} A_{2}^{n}\left(I-A_{2} A_{2}^{d}\right) \\
& -\left[I-\left(A_{1}-H_{1}\right)\left(A_{1}-H_{1}\right)^{d}\right] \sum_{n=0}^{\infty}\left(A_{1}-H_{1}\right)^{n} H_{2}\left(A_{2}^{d}\right)^{n+2} .
\end{aligned}
$$

Notice that $R^{d}=\left[\begin{array}{cc}\left(A_{1}-H_{1}\right)^{d} & 0 \\ 0 & 0\end{array}\right]$. Now a straightforward computation shows:

$$
\begin{aligned}
& \left(I-R R^{d}\right)(A-H)^{n} H(I-F)\left(A^{d}\right)^{n+2} \\
& \quad=\left[\begin{array}{lc}
0 & {\left[I-\left(A_{1}-H_{1}\right)\left(A_{1}-H_{1}\right)^{d}\right]\left(A_{1}-H_{1}\right)^{n} H_{2}\left(A_{2}^{d}\right)^{n+2}} \\
0 & 0
\end{array}\right], \\
& \left(I+R^{d} H\right)(I-F) A^{d}=\left[\begin{array}{cc}
0 & \left(A_{1}-H_{1}\right)^{d} H_{2} A_{2}^{d} \\
0 & A_{2}^{d}
\end{array}\right]
\end{aligned}
$$

and

$$
\left(R^{d}\right)^{n+2} H(I-F) A^{n}\left(I-A A^{d}\right)=\left[\begin{array}{cc}
0 & \left(\left(A_{1}-H_{1}\right)^{d}\right)^{n+2} H_{2} A_{2}^{n}\left(I-A_{2} A_{2}^{d}\right) \\
0 & 0
\end{array}\right] .
$$

Hence, the proof is completed. 
Campbell and Meyer (see [2]) investigated the continuity properties of the Drazin inverse of complex square matrices, but they did not establish the norm estimates for the perturbation of the Drazin inverse. Their results are extended to infinite dimensional settings by Rakočević (see [4]) and Koliha and Rakočević (see [15]), but norm estimates are not established there. It is interesting to mention that special cases of these perturbation results are already known. For example, see [20] for complex square matrices, [5] for complex Banach algebras and [8] for unbounded operators on Banach spaces. See also Case (4) of the following section. Hence, in this paper we partially solve the previous problem of Campbell and Meyer and extend some well-known results from previous papers.

\section{Special cases}

Many interesting special cases of our Theorem 2.4 are considered in [13] for matrices. Some of them are generalizations of well-known results.

Case (1). If $H F=0$, then $(A-H)^{n} F H=A^{n} H$ for $n \geq 0$. Consequently,

$$
\begin{aligned}
(A-H)^{d}= & A^{d} F-\sum_{n=0}^{\infty}\left(A^{d}\right)^{n+2} H A^{n}\left(I-A A^{d}\right) \\
& +\left(I-F+A^{d} H\right) A^{d}-\sum_{n=0}^{\infty} A^{n}\left(I-A A^{d}\right) H\left(A^{d}\right)^{n+2} .
\end{aligned}
$$

Case (1a). If $H F=0$ and $F=A A^{d}$, then $H A^{d}=H A A^{d}=0$ and $(A-H)^{d}=$ $A^{d}-\sum_{n=0}^{\infty}\left(A^{d}\right)^{n+2} H A^{n}$.

Case (1b). If $H F=0$ and $F=I-A A^{d}$, then $A^{d} H=0$ and $(A-H)^{d}=$ $A^{d}-\sum_{n=0}^{\infty} A^{n} H\left(A^{d}\right)^{n+2}$.

Case (2). If $F=A A^{d}$, then $(A-H)^{d}=R^{d}-\sum_{n=0}^{\infty}\left(R^{d}\right)^{n+2} H A^{n}\left(I-A A^{d}\right)$.

Case (2a). Let $F=A A^{d}$ and let $U=I-A^{d} H A A^{d}$ be invertible. Recall notation from Theorem 2.4, noticing that $A_{1}$ is invertible and $A_{2}$ is quasinilpotent. Then $U=\left[\begin{array}{cc}I-A_{1}^{-1} H_{1} & 0 \\ 0 & I\end{array}\right]$. Since $U$ is invertible, then $I-A_{1}^{-1} H_{1}$ is invertible and $A_{1}-H_{1}=$ $A_{1}\left(I-A_{1}^{-1} H_{1}\right)$ is invertible. Hence, $\operatorname{ind}(R) \leq 1$,

$$
R^{*}=\left[\begin{array}{cc}
\left(I-A_{1}^{-1} H_{1}\right)^{-1} A_{1}^{-1} & 0 \\
0 & 0
\end{array}\right]
$$

and $(A-H)^{d}=R^{*}-\sum_{n=0}^{\infty}\left(R^{*}\right)^{n+2} H A^{n}\left(I-A A^{d}\right)$.

Let $V=I-A A^{d} H A^{d}=\left[\begin{array}{cc}I-H_{1} A_{1}^{-1} & 0 \\ 0 & I\end{array}\right]$ and $W=I-A^{d} H=\left[\begin{array}{cc}I-A_{1}^{-1} H_{1}-A_{1}^{-1} H_{2} \\ 0 & I\end{array}\right]$. Notice that $U$ is invertible if and only if $1 \notin \sigma\left(A_{1}^{-1} H_{1}\right)$, if and only if $1 \notin \sigma\left(H_{1} A_{1}^{-1}\right)$, since it is well-known that for $T \in \mathscr{L}(X, Y)$ and $S \in \mathscr{L}(Y, X)$

$$
\sigma(S T) \cup\{0\}=\sigma(T S) \cup\{0\} .
$$


Hence, $U$ is invertible if and only if $V$ is invertible if and only if $W$ is invertible. We also easily verify $R^{*}=U^{-1} A^{d}=A^{d} V^{-1}$. Hence, this result generalizes the main results in $[16,18,20,19]$.

Case (2b). If $F=I-A A^{d}$, then $A^{d} H=A^{d} F=0$,

$$
R=A\left(I-A A^{d}\right)-\left(I-A A^{d}\right) H\left(I-A A^{d}\right)
$$

and $(A-H)^{d}=R^{d}+\left(I+R^{d} H\right) A^{d}-\left(I-R R^{d}\right) \sum_{n=0}^{\infty}(A-H)^{n} H\left(A^{d}\right)^{n+2}$.

Case (3). Let $A A^{d} H F=H F A A^{d}=H F$, let $U=I-A^{d} H F$ be invertible and $(A F)^{*}$ exists. Recall notation from Theorem 2.4. Then $A F=\left[\begin{array}{cc}A_{1} & 0 \\ 0 & 0\end{array}\right]$, implying $\operatorname{ind}\left(A_{1}\right) \leq 1$. From $A A^{d} H F=H F A A^{d}=H F$ we conclude $A_{1} A_{1}^{*} H_{1}=H_{1} A_{1} A_{1}^{\prime \prime}=H_{1}$. We also have

$$
U=\left[\begin{array}{cc}
I-A_{1}^{*} H_{1} & 0 \\
0 & I
\end{array}\right]
$$

Let

$$
V=I-H F A^{d}=V=\left[\begin{array}{cc}
I-H_{1} A_{1}^{*} & 0 \\
0 & I
\end{array}\right] .
$$

By (3) we conclude that $U$ is invertible if and only if $V$ is invertible. Now a matrix computation shows $R=A F U=V F A$. From $A_{1}^{*}\left(I-H_{1} A_{1}^{*}\right)=\left(I-A_{1}^{*} H_{1}\right) A_{1}^{*}$ we get the equality $A^{d} F V=U A^{d} F$. Let $Y=U^{-1} A^{d} F=A^{d} F V^{-1}$. Then it is easy to verify $Y=R^{n}$. Finally, we have $(A-H)^{d}=R^{*}-\sum_{n=0}^{\infty}\left(R^{n}\right)^{n+2} H\left(I-A A^{d}\right) A^{n}$. Case (4). If $F H=H F=F$, then $(A-H)^{d}=R^{d}+(I-F) A^{d}$. Moreover, if $F=A A^{d}$ and $U=I-A^{d} H$ is invertible, then $(A-H)^{d}=R^{d}=U^{-1} A^{d}$.

Case (4) shows that results of this paper are more general than the corresponding results in [20]. Analogous results are proved in complex Banach algebras in [5]. Results of this paper are more general then results in [5] if we consider a Banach algebra of all operators on a fixed complex Banach space. We expect that all results of this paper should be valid in an arbitrary complex Banach algebra with a unit, but this will be a matter of further investigations. It is interesting to mention results related to this Case (4), concerning the Drazin inverse for closed linear operators, as it is done in [8].

\section{References}

[1] A. Ben-Israel and T. N. E. Greville, Generalized inverses: theory and applications (Wiley-Interscience, New York, 1974).

[2] S. L. Campbell and C. D. Meyer, Generalized inverses of linear transformations (Pitman, New York, 1979). 
[3] S. R. Caradus, Generalized inverses and operator theory, Queen's Papers in Pure and Appl. Math. 50 (Queen's University, Kingston, Ontario, 1978).

[4] V. Rakočević, 'Continuity of the Drazin inverse', J. Operator Theory 41 (1999), 55-68.

[5] V. Rakočević and Y. Wei, 'The perturbation theory for the Drazin inverse and its applications II', J. Austral. Math. Soc. 70 (2001), 189-197.

[6] D. S. Djordjević and P. S. Stanimirović, 'On the generalized Drazin inverse and generalized resolvent', Czechoslovak Math. J. 51 (126) (2001), 617-634.

[7] M. P. Drazin, 'Pseudoinverses in associative rings and semigroups', Amer. Math. Monthly 65 (1958), 506-514.

[8] N. Castro González and J. J. Koliha, 'Perturbation of the Drazin inverse for closed linear operators', Integral Equations Operator Theory 36 (2000), 92-106.

[9] R. E. Harte, 'Spectral projections', Irish Math. Soc. Newsletter 11 (1984), 10-15.

[10] _ Invertibility and singularity for bounded linear operators (Marcel Dekker, New York, 1988).

[11] _ 'On quasinilpotents in rings', Panamer. Math. J. 1 (1991), 10-16.

[12] R. E. Hartwig and J. M. Shoaf, 'Group inverses and Drazin inverses of bidiagonal and triangular Toeplitz matrices', J. Austral. Math. Soc. Ser. A 24 (1977), 10-34.

[13] R. E. Hartwig, G. Wang and Y. Wei, 'Some additive results on Drazin inverse', Linear Algebra Appl. 322 (2001), 207-217.

[14] J. J. Koliha, 'A generalized Drazin inverse', Glasgow Math. J. 38 (1996), 367-381.

[15] J. J. Koliha and V. Rakočević, 'Continuity of the Drazin inverse II', Studia Math. 131 (1998), $167-177$.

[16] C. D. Meyer, Jr., 'The condition number of a finite Markov chains and perturbation bounds for the limiting probabilities', SIAM J. Algebraic Discrete Methods 1 (1980), 273-283.

[17] C. D. Meyer, Jr. and N. J. Rose, 'The index and the Drazin inverse of block triangular matrices', SIAM J. Appl. Math. 33 (1977), 1-7.

[18] C. D. Meyer, Jr. and J. M. Shoaf, 'Updating finite Markov chains by using techniques of group inversion', J. Statist. Comput. Simulation 11 (1980), 163-181.

[19] Y. Wei, 'On the perturbation of the group inverse and the oblique projection', Appl. Math. Comput. 98 (1999), 29-42.

[20] Y. Wei and G. Wang, 'The perturbation theory for the Drazin inverse and its applications', Linear Algebra Appl. 258 (1997), 179-186.

Department of Mathematics

Faculty of Sciences

University of Niš

P.O. Box 224

18000 Niš

Yugoslavia

e-mail: dragan@pmf.pmf.ni.ac.yu
Department of Mathematics and Laboratory of Mathematics for Nonlinear Sciences Fudan University Shanghai 200433 P.R. of China e-mail: ymwei@fudan.edu.cn 
